\title{
Structure and embedding theorems for unique normal decomposition lattices
}

by

E. W. Johnson (Iowa City, Ia.), J. A. Johnson (Houston, Tex.), and J. P. Lediaev (Iowa City, Ia.)

In [2] we characterized those distributive Noether lattices which can be represented as the lattice of ideals of a Noetherian ring. Those lattices have the property that every element has a unique normal primary decomposition involving only powers of primes. In this paper we consider a broader class of multiplicative lattices, namely those which satisfy the less restrictive condition that every element has a unique normal decomposition. For such a lattice $\mathcal{L}$ we obtain a structure theorem (Theorem 1), and a characterization in terms of a "dense" embedding of the lattice of ideals of a suitable Noetherian ring (Theorem 2). We also give a condition under which $\mathfrak{L}$ can be represented as the lattice of ideals of some Noetherian ring (Theorem 3).

A (commutative) multiplicative lattice is a complete lattice in which there is defined a commutative, associative and join-distributive multiplication for which the greatest element, denoted by $I$, is the multiplicative identity. An element $M$ in a multiplicative lattice $\mathcal{L}$ is a join-principal element if $(A \vee B M): M=A: M \vee B$ for all $A, B \in \mathcal{L}$. Each principal ideal in a commutative ring $R$ is a join-principal element in the lattice of ideals of $R$ [1].

Throughout this paper, $\mathfrak{L}$ will denote a commutative multiplicative lattice in which each element is a join (not necessarily finite) of join-principal elements and in which $I$ is compact.

An element $Q$ is primary for a prime element $P$ (or $Q$ is $P$-primary) if $P^{n} \leqslant Q \leqslant P$ for some integer $n$ and the relation $A B \leqslant Q$ implies that $A \leqslant Q$ or $B \leqslant P$. An irredundant decomposition $A=Q_{1} \wedge \ldots \wedge Q_{k}$ is a normal decomposition of $A$ if $Q_{i}$ is primary for, say, $P_{i}$ and the $P_{i}$ are distinct. $\mathcal{L}$ is a unique normal decomposition lattice (or UND lattice) if each element of $\mathcal{L}$ has a unique normal decomposition.

We will first prove the Krull Intersection Theorem for $\mathfrak{L}$. 
LEMMA 1. Let $\mathfrak{L}$ be a lattice in which each element has a normal decomposition. Let $g$ denote the greatest lower bound of the collection of maximal elements of $\mathcal{L}$, and let $B$ be an element of $\mathcal{L}$ such that $B \leqslant \mathcal{\gamma}$. Then $\bigwedge_{k=1}^{\infty}\left(A \vee B^{k}\right)$ $=A$ for each $A \in \mathcal{L}$.

Proof. It suffices to prove that if $M$ is a join-principal element such that $M \leqslant \bigwedge_{k=1}^{\infty}\left(A \vee B^{k}\right)$, then $M \leqslant A$. Hence, let $M$ be a join-principal element which satisfies the relation $M \leqslant \bigwedge_{k=1}^{\infty}\left(A \vee B^{k}\right)$. Let $A=Q_{1} \wedge \ldots \wedge Q_{n}$ be a normal decomposition of $A$ where $Q_{i}$ is $P_{i}$-primary, and let $A \vee B M$ $=T_{1} \wedge \ldots \wedge T_{m}$ be a normal decomposition of $A \vee B M$ where $T_{i}$ is $S_{i}$-primary. Since $T_{i}$ is $S_{i}$-primary, we have that for each $i(1 \leqslant i \leqslant m)$, either $M \leqslant T_{i}$ or $B^{k_{i}} \leqslant T_{i}$ for some integer $k_{i}$. In the latter case the relations $T_{i} \geqslant A \vee B^{k_{i}}$ $\geqslant \bigwedge_{k=1}^{\infty}\left(A \vee B^{k}\right) \geqslant M$ hold. Thus, in either case $M \leqslant T_{i}$ for each $i$, and so $M \leqslant A \vee B M$ and $A \vee M=A \vee B M$. Since $M$ is join-principal, we have $A: M \vee B=(A \vee B M): M=(A \vee M): M=I$. From this and from the relation $B \leqslant \xi$, we conclude that $A: M \nless P_{i}$ for each $i=1, \ldots, n$. Since (A: $M) M \leqslant A \leqslant Q_{i}$, we have $M \leqslant Q_{i}$ for each $i=1, \ldots, n$. Therefore, $M \leqslant A$.

LemMa 2. If $D$ and $M$ are elements of $\mathcal{L}$ such that $M^{m} \leqslant D \leqslant M$ for some integer $m$ and if $M$ is a maximal element in $\mathcal{L}$, then $D$ is $M$-primary.

Proof. Suppose that $A B \leqslant D$ but $B \nless M$. Then $(M \vee B)^{m}=I$, hence $A=A(M \vee B)^{m}=A M^{m} \vee A B M^{m-1} \vee \ldots \vee A B^{m-1} M \vee A B^{m}$. Since $M^{m} \leqslant D$ and $A B \leqslant D$, it follows that $A \leqslant D$.

If $\mathcal{L}$ has only one maximal element, $\mathcal{L}$ is quasi-local. If $\mathcal{L}$ has only one prime element, $\mathcal{L}$ is primary. $\mathcal{L}$ is said to be one-dimensional if there exists at least one pair of distinct primes which are comparable but no three distinct primes are pairwise comparable. The following lemma, which extends a known ring theoretic result to multiplicative lattices, classifies the quasi-local UND lattices.

LEMMA 3. If $\mathcal{L}$ is a quasi-local $U N D$ lattice, then $\mathcal{L}$ is either primary or $\mathcal{L}$ is a one-dimensional lattice in which 0 is prime.

Proof. Assume that $\mathcal{L}$ is not a primary lattice. Let $M$ be the maximal element of $\mathcal{L}$ and let $P$ be a nonmaximal prime. Let $P M=Q_{1} \wedge \ldots \wedge Q_{k}$ be the normal decomposition of $P M$ where $Q_{i}$ is $P_{i}$-primary. Then for each $i$, we have $P M \leqslant Q_{i}$, so either $P \leqslant Q_{i}$ or $M \leqslant P_{i}$. Suppose that $M \leqslant P_{i}$ for some $i$, say $M \leqslant P_{1}$. Then $Q_{1}$ is $M$-primary, so there is an integer $n$ such that $M^{n} \leqslant Q_{1}$. It follows that $\left(M^{r} \vee P M\right) \wedge Q_{2} \wedge \ldots \wedge Q_{k}$ is a normal decomposition of $P M$ for all $r \geqslant n$, and hence that $Q_{1}$ $\leqslant \bigwedge_{r=1}^{\infty}\left(M^{r} \vee P M\right)$. But then, by Lemma $1, M^{n} \leqslant Q_{1} \leqslant P M \leqslant P$, which contradicts the fact that $M \neq P$. Hence $M \nless P_{i}$ for all $i$, so $P \leqslant Q_{i}$ for all $i$. It now follows that $P \leqslant P M$, and hence that $P \leqslant \bigwedge_{n=1}^{\infty} M^{n}=0$. Since $P$ was an arbitrary nonmaximal prime in $\mathcal{L}$, this completes the proof of the lemma.

For an arbitrary element $D$ in $\mathcal{L}$, let $\mathcal{L} / D$ denote the sublattice of $\mathcal{L}$ which consists of all elements $A$ in $\mathcal{L}$ that satisfy the relation $A \geqslant D$. For $A, B \in \mathcal{L} / D$, define $A \circ B=A B \vee D$. With this multiplication, $\mathcal{L} / D$ is a commutative multiplicative lattice in which each element is a join of join-principal elements in $\mathfrak{L} / D$ [1].

Suppose that each element of $\mathcal{L}$ has a normal decomposition and let $A=Q_{1} \wedge \ldots \wedge Q_{n}$ be a normal decomposition of $A \in \mathcal{L}$ where $Q_{i}$ is $P_{i}$-primary. If $D$ is an arbitrary element of $\mathcal{L}$, then $\left\{P_{i} \mid P_{i} \vee D \neq I\right\}$ is an isolated set of primes of $A$. Let $A_{D}$ denote the corresponding isolated component of $A$ (i.e., $A_{D}=\bigwedge\left\{Q_{i} \mid P_{i} \vee D \neq I\right\}$ ), and define $A \equiv B(D)$ if and only if $A_{D}=B_{D}$. Dilworth [1] proved that the congruence $\bmod D$ is a congruence relation on $\mathcal{L}$ which preserves meet, join, multiplication, and residuation. Let $\mathscr{L}_{D}$ denote the multiplicative lattice of congruence classes. For each element $A \in \mathcal{L}$, we let $\{A\}$ denote the congruence class of $A$. Since join-principal elements are defined in terms of an equation involving join, multiplication, and residuation, it follows that the congruence class of a join-principal element in $\mathcal{L}$ is join-principal in $\mathcal{L}_{D}$. Therefore each element in $\mathfrak{L}_{D}$ is a join of join-principal elements. The primes and primaries of $\mathfrak{L}_{D}$ are precisely the congruence classes determined by the primes and primaries of $\mathcal{L}$. We are now ready to prove the following structure theorem for UND lattices.

THEOREM $1 . \mathcal{L}$ is a UND lattice if and only if $\mathfrak{L}$ is a finite direct sum of primary lattices having nilpotent maximal elements and one-dimensional lattices in which 0 is prime and in which each nonzero element is greater than or equal to a product of nonzero prime elements.

Proof. Assume that $\mathcal{L}$ is a UND lattice. Let $0=Q_{1} \wedge Q_{2} \wedge \ldots \wedge Q_{n} \wedge$ $\wedge P_{1} \wedge \ldots \wedge P_{m}$ be the unique normal decomposition of 0 where each $Q_{i}$ is primary for a maximal element, say $M_{i}$, and each $P_{j}$ is primary for a nonmaximal prime element. Let $M$ be any maximal element of $\mathcal{L}$ such that $M \geqslant P_{j}$ for some $j$, and let $P_{j}$ be $P$-primary. Since the UND property of $\mathcal{L}$ is inherited by $\mathcal{L}_{M}, \mathfrak{L}_{M}$ is a one-dimensional lattice in which $\{0\}$ is prime (Lemma 3). Therefore the primes $\{0\},\{P\}$, and $\{M\}$ cannot be distinct and so $\{0\}=\{P\}$ (since $\{P\} \neq\{M\}$ ). Consequently $P=P_{M}$ $=0_{M} \leqslant P_{j} \leqslant P$ and so $P_{j}$ is prime. Again by one-dimensionality of $\mathfrak{L}_{M}$ we also conclude that $P_{j}$ is the only prime element in $\mathcal{L}$ such that $P_{j} \leqslant M$. Thus if $i \neq j$, then $P_{i}$ and $P_{j}$ are comaximal primes. Since the $Q_{j}$ are primary for maximal elements, they are pairwise comaximal. Furthermore, we claim that any pair $P_{i}, Q_{k}$ is comaximal. For suppose $P_{i} \vee Q_{k} \neq I$, 
and let $M$ be a maximal element such that $P_{i} \vee Q_{k} \leqslant M$. From $\{0\}$ $=\left\{P_{i}\right\} \leqslant\left\{Q_{k}\right\}$ we conclude that $\left\{P_{i}\right\}=\left\{P_{i}\right\} \wedge\left\{Q_{k}\right\}=\left\{P_{i} \wedge Q_{k}\right\}$, hence $P_{i}=\left(P_{i}\right)_{M}=\left(P_{i} \wedge Q_{k}\right)_{M}=P_{i} \wedge Q_{k}$. This contradicts the irredundancy of the given normal decomposition of 0 . Therefore the elements in $\left\{Q_{1}, \ldots, Q_{n}, P_{1}, \ldots, P_{m}\right\}$ are pairwise comaximal and $\mathcal{L} \cong \mathcal{L} / Q_{1} \oplus \ldots \oplus$ $\oplus \mathcal{L} / Q_{n} \oplus \mathcal{L} / P_{1} \oplus \ldots \oplus \mathcal{L} / P_{m}$.

For each $Q_{i}, \mathcal{L} / Q_{i}$ is clearly a primary lattice in which the maximal element is nilpotent. For each $j, \mathcal{L} / P_{j}$ is one-dimensional since if $M$ is a maximal element of $\mathcal{L}$ such that $M \geqslant P_{j}$, then $\mathfrak{L}_{M}$ is one-dimensional by an above argument. Since each element of $\mathcal{L} / P_{j}$ has a normal decomposition, it is clear that each nonzero element of $\mathcal{L} / P_{j}$ is greater than or equal to a product of nonzero prime elements. This completes the proof of the "only if" part of the theorem.

We will now prove that a finite direct sum of lattices which satisfy the conditions stated in the theorem is a UND lattice. Olearly a primary lattice having a nilpotent maximal elements is a UND lattice since each element of such a lattice is primary for the maximal element. Also, the direct sum of UND lattices is clearly a UND lattice, so we need only prove that a one-dimensional lattice in which zero is prime, and in which every nonzero element is greater than or equal to a product of nonzero prime elements, is a UND lattice. Let $\mathcal{L}$ be such a lattice. Let $A$ be a nonzero element of $\mathcal{L}$, and let $P_{1}, \ldots, P_{n}$ be distinct nonzero primes such that $P_{1}^{k_{1}} \ldots P_{n}^{k_{n}} \leqslant A$ for some positive integers $k_{i}$. If $M$ is a maximal element such that $M \geqslant A$, then $M \geqslant P_{i}$ for some $i$ (since $M$ is prime) and so $M=P_{i}$. Thus there are only a finite number of maximal elements which are greater than or equal to $A$ and they are among the $P_{i}$. Let $\left\{M_{1}, \ldots, M_{s}\right\}$ be the collection of all distinct maximal elements such that $M_{i} \geqslant A$. For each $M_{i}$, define $F_{i}=\{B \in \mathcal{L} \mid$ there exists an element $T \in \mathcal{L}$ such that $T \leqslant M_{i}$ and $\left.B T \leqslant A\right\}$, and define $Q_{i}=\bigvee_{B \in F_{i}} B$. Clearly $A \leqslant Q_{i}$ (take $B=A$ and $T=I$ ). We will now prove that $Q_{i}$ is $M_{i}$-primary. If $B$ is an element such that $B T \leqslant A$ for some element $T \nless M_{i}$, then, since $M_{i}$ is prime and $B T \leqslant M_{i}$, we have $B \leqslant M_{i}$; consequently $Q_{i} \leqslant M_{i}$. Since $M_{i}$ is one of the $P_{j}$, say $M_{i}=P_{1}$, and since the $P_{j}$ are distinct, we conclude that $M_{i}^{k_{1}} T \leqslant A$ but $T \nless M_{i}$ where $T=P_{2}^{k_{2}} \ldots P_{n}^{k_{n}}$. By definition of $Q_{i}$ it follows that $M_{i}^{k_{1}} \leqslant Q_{i}$ and so $Q_{i}$ is $M_{i}$-primary (Lemma 2). Let $D=Q_{1} \wedge \ldots \wedge Q_{s}$ and observe that $A \leqslant D$. We will now show that $D \leqslant A$. Since the $Q_{i}$ are primary for (distinct) maximal elements, they are pairwise comaximal, hence $D=Q_{1} \wedge \ldots \wedge Q_{s}=Q_{1} Q_{2} \ldots Q_{s}$. Consequently

$$
D=\left(\bigvee_{B \in F_{1}} B\right) \ldots\left(\bigvee_{B \in F_{s}} B\right)=\bigvee\left\{B_{1} \ldots B_{s} \mid B_{i} \in F_{i} \text { for each } i=1, \ldots, s\right\}
$$

where the last equality holds since multiplication distributes over arbitrary joins. Let $B=B_{1} \ldots B_{s}$ be an arbitrary product where $B_{i} \in F_{i}$.
Then, for each $i$, there exists an element $T_{i} \nless M_{i}$ such that $B_{i} T_{i} \leqslant A$. If $M$ is a maximal element of $\mathcal{L}$ such that $M$ is not one of the $M_{j}$, then $A: B \leqslant M$ (otherwise $A \leqslant A: B \leqslant M$ ). If $M=M_{j}$ for some $j$, then the relations $B T_{j} \leqslant B_{j} T_{j} \leqslant A$ and $T_{j} \leqslant M_{j}$ imply that $A: B \leqslant M_{j}$ (otherwise $T_{j} \leqslant A: B \leqslant M_{j}$ ). Thus $A: B$ is not less than or equal to any maximal element of $\mathcal{L}$, so $A: B=I$ and $B \leqslant A$. Consequently $A=D$ and $A$ has a normal decomposition. Since $A$ has no embedded primes, this normal decomposition of $A$ is unique. This completes the proof of the theorem.

Let $\mathcal{L}$ and $\mathcal{L}^{\prime}$ be multiplicative lattices such that each element of $\mathcal{L}^{\prime}$ has a normal decomposition. A one-to-one function $f$ mapping $\mathcal{L}$ into $\mathcal{L}^{\prime}$ is a multiplicative lattice embedding if it preserves meets, joins, and products, and if it maps primes into primes, primaries into primaries, and 0 into 0 . If $f$ also has the property that for each element $A^{\prime} \in \mathcal{L}^{\prime}$ which is not less than or equal to any isolated component of zero there exists a nonzero $A \in \mathcal{L}$ such that $f(A) \leqslant A^{\prime}$, then $f$ is called a dense multiplicative lattice embedding.

THEOREM 2. Let $\mathcal{L}$ be a lattice in which each element has a normal decomposition. $\mathcal{L}$ is a $\mathrm{UND}$ lattice if and only if there exists a dense embedding of a lattice of ideals of a finite direct sum of Dedekind domains and homomorphic images of regular local rings of altitude one into $\mathfrak{L}$ such that maximal ideals are mapped onto maximal elements of $\mathfrak{L}$.

Proof. Let $\mathcal{L}$ be a UND lattice. By Theorem $1, \mathcal{L}$ is a finite direct $\operatorname{sum} \mathfrak{L} \cong \mathfrak{L}_{1} \oplus \ldots \oplus \mathcal{L}_{n} \oplus \mathcal{L}_{n+1} \oplus \ldots \oplus \mathcal{L}_{m}$ of lattices, where for each $i=1, \ldots, n$, $\mathcal{L}_{i}$ is a primary lattice having a nilpotent maximal element $N_{i}$, and for each $i=n+1, \ldots, m, \mathfrak{L}_{i}$ is a one-dimensional lattice in which 0 is prime and each nonzero element of $\mathcal{L}_{i}$ is greater than or equal to a product of nonzero prime elements. Let $R$ be a regular local ring of altitude one and let $N$ denote its maximal ideal. Each non-zero ideal of $R$ is a power of $N$ [3]. For each $i=1, \ldots, n$, let $k_{i}$ be the least positive integer such that $N_{i}^{k_{i}}=0$, let $L_{i}$ denote the lattice of ideals of $R / N^{k i}$, let $M_{i}$ denote the maximal element of $L_{i}$, and define $f_{i}: L_{i} \rightarrow L_{i}$ by defining $f_{i}\left(M_{i}^{k}\right)=N_{i}^{k}$. clearly $f_{i}$ is a dense embedding.

Now fix an integer $i=n+1, \ldots, m$ and let $\mu \mathfrak{L}_{i}=\left\{M_{1}^{k_{1}} \ldots M_{m}^{k_{m}} \mid M_{j}\right.$ is a maximal element of $\mathcal{L}_{i}$ and $\left.k_{j} \geqslant 0\right\} \cup\{0\}$. Since $\mathcal{L}_{i}$ is one-dimensional, $\mu \complement_{i}$ is a distributive sublattice of $\mathcal{L}_{i}$ in which each element is a unique product of prime elements and in which 0 is prime. Furthermore, for distinct nonzero primes (hence maximal) $M_{j}$ of $\mu \mathfrak{L}_{i}$ and for integers $e_{j}, f_{j} \geqslant 0$, we have the following:

$$
\left(\prod_{1}^{n} M_{j}^{e_{j}}\right) \cdot\left(\prod_{1}^{n} M_{j}^{f_{j}}\right)=\prod_{1}^{n} M_{j}^{e_{j}+f_{j}}
$$




$$
\begin{aligned}
\left(\prod_{1}^{n} M_{j}^{e_{j}}\right) \wedge\left(\prod_{1}^{n} M_{j}^{f_{j}}\right) & =\left(\bigwedge_{1}^{n} M_{j}^{e_{j}}\right) \wedge\left(\bigwedge_{1}^{n} M_{j}^{f_{j}}\right) \\
& =\bigwedge_{1}^{n} M_{j}^{\max \left(e_{j}, f_{j}\right)}=\prod_{1}^{n} M_{j}^{\max \left(e_{j}, f_{j}\right)}
\end{aligned}
$$

and

$$
\begin{aligned}
\left(\prod_{1}^{n} M_{j}^{e_{j}}\right) \vee\left(\prod_{1}^{n} M_{j}^{t_{j}}\right) & =\left(\bigwedge_{1}^{n} M_{j}^{e_{j}}\right) \vee\left(\bigwedge_{1}^{n} M_{j}^{f_{j}}\right) \\
& =\bigwedge_{1}^{n} M_{j}^{\min \left(e_{j}, f_{j}\right)}=\prod_{1}^{n} M_{j}^{\min \left(e_{j}, f_{j}\right)}
\end{aligned}
$$

We will now construct a Dedekind domain whose lattice of ideals can be densely embedded in $\mathfrak{L}_{i}$. Let $\alpha$ be the cardinality of the collection of maximal primes in $\mathcal{L}_{i}$, and let $K$ be a field of cardinality $\beta \geqslant \alpha$. Let $A$ be a subset of $K$ of cardinality $\alpha$, and let $S$ be the compliment in $K[x]$ of the union of the prime ideals $(a+x), a \in A$. Then $S$ is a multiplicatively closed subset of $K[x]$ which doesn't meet any of the prime ideals $(a+x)$, and which meets every other prime ideal. Hence $D_{i}=K[x]_{S}$ is a Dedekind domain with a maximal prime ideals [4]. Let $L_{i}$ denote the lattice of ideals of $D_{i}$. Let $f_{i}^{\prime}$ be a one-to-one correspondence between the maximal primes of $L_{i}$ and the maximal primes of $\mathcal{L}_{i}$, and extend $f_{i}^{\prime}$ to a map $f_{i}$ of $L_{i}$ onto $\mu \mathfrak{L}_{i}$ by taking 0 to 0 and products to products. Since $L_{i}$ also satisfies the above properties (a), (b) and (c), it follows that $f_{i}$ is an isomorphism of $L_{i}$ onto $\mu \mathcal{L}_{i}$, and hence an embedding into $\mathcal{L}_{i}$. Since each nonzero element of $\mathfrak{L}_{i}$ is greater than or equal to a product of maximal elements, $f_{i}$ is dense. Therefore there is a dense embedding of $L_{1} \oplus \ldots \oplus L_{m}$ into $\mathfrak{L}_{1} \oplus \ldots \oplus \mathfrak{L}_{m}$. This completes the proof of the "only if" part of the theorem.

Conversely, let $L$ be the lattice of ideals of a ring satisfying the conditions stated in the theorem, and let $f$ be a dense embedding of $L$ into $\mathcal{L}$ such that $f$ maps the maximals in $L$ onto the maximals of $\mathcal{L}$. The function $f$ maps normal decomposition of 0 in $L$ to a normal decomposition of 0 in $\mathcal{L}$. Let $0=R_{1} \wedge \ldots \wedge R_{r}$ be this normal decomposition of 0 in $\mathcal{L}$ where $R_{i}$ is $P_{i}$-primary. If $R_{i} \leqslant P_{i}$, then $P_{i}$ is not less than or equal to any isolated component of 0 , and so there exists a nonzero element $B \in L$ such that $f(B) \leqslant P_{i}$. Since $B$ is a product of maximal elements in $L, P_{i}$ is greater than or equal to a product of maximal elements in $\mathfrak{L}$ and thus $P_{i}$ is maximal in $\mathcal{L}$. Therefore, each $R_{i}$ is either prime or is a primary for a maximal element. Consequently, the above normal decomposition of 0 is unique. From this and from the properties of $f$ we conclude that if $D$ is less than or equal to an isolated component of 0 , then $D$ also has a unique normal decomposition. If $A$ is not less than or equal to any isolated component of zero, then $A$ is greater than or equal to a product of maximal elements of $\mathcal{L}$ (since $f$ is a dense embedding), and so $A$ is less than or equal to only finitely many distinct maximal elements $M_{i}(1 \leqslant i \leqslant n)$ in $\mathcal{L}$. For each $M_{i}$, we construct a primary component $Q_{i}$ of $A$ as we did in the proof of Theorem 1. Then $A=Q_{1} \wedge \ldots \wedge Q_{n}$ is the unique normal decomposition of $A$. Therefore $\mathcal{L}$ is a UND lattice,

THEOREM 3. Let $\mathcal{L}$ be a $U N D$ lattice. Each primary element of $\mathcal{L}$ is a power of a prime if and only if $\mathcal{L}$ is represented as a lattice of ideals of a direct sum of Dedekind domains and homomorphic images of regular local rings of altitude one.

Pro of. By Theorem $1, \mathcal{L}$ is a finite direct sum $\mathcal{L} \cong \mathfrak{L}_{1} \oplus \ldots \oplus \mathfrak{L}_{n} \oplus \ldots \oplus \mathcal{L}_{m}$ of lattices, where for each $i=1, \ldots, n, \mathcal{L}_{i}$ is a primary lattice having a nilpotent maximal element $N_{i}$, and for each $i=n+1, \ldots, m, \mathfrak{L}_{i}$ is a one-dimensional lattice in which 0 is prime and each nonzero element of $\mathcal{L}_{i}$ is greater than or equal to a product of nonzero prime elements. If the primaries of $\mathcal{L}$ are powers of primes, then $\mathcal{L}_{i}=\left\{N_{i}^{k}\right\} k=1, \ldots, s_{i}$ where $s_{i}$ is the least positive integer such that $\left.N_{i}^{s_{i}}=0\right\}$ for $i=1, \ldots, n$, and $\mathfrak{L}_{i}=\mu \mathfrak{L}_{i}$ for $i=n+1, \ldots, m$ : Consequently the embedding in the proof of Theorem 1 is an isomorphism onto $\mathcal{L}$. The converse of the theorem is clear.

\section{References}

[1] R. P. Dilworth, Abstract commutative ideal theory, Pacific J. Math. 12 (1962), pp. 481-498.

[2] E.W. Johnson and J.P. Lediaev, Representable distributive Noether lattices, Pacific J. Math. 28 (1969), pp. 561-564.

[3] D. G. Northeott, Ideal Theory, Cambridge 1963.

[4] O. Zarski and P. Samuel, Commutative Algebra, Vol. 1, Princeton 1960.

THE UNIVERSITY OF IOWA

Iowa City, Iowa

Iowa
and
CALI

CALIFORNIA INSTITUTE OF TECHNOLOGY

TET PROPULSION LAB

and THE UNIVERSITY OF HOUSTON

Reģu par la Rédaction le 22. 8. 1969 